\title{
Novel Approaches to Assessing Cardiac Safety - Proceedings of a Workshop
} Regulators, Industry and Academia Discuss the Future of In Silico Cardiac Modelling to Predict the Proarrhythmic Safety of Drugs

\author{
Katherine Fletcher, ${ }^{1}$ Rashmi R. Shah, ${ }^{2,3}$ Arthur Thomas, ${ }^{2,4}$ Frank Tobin ${ }^{2,5}$ Blanca Rodriguez, ${ }^{1}$ \\ Gary R. Mirams, ${ }^{1}$ Javier Saiz ${ }^{6}$ and Denis Noble, ${ }^{1}$ on behalf of the preDiCT Project \\ 1 University of Oxford, Oxford, UK \\ 2 Member of the preDiCT Scientific Advisory Board, University of Oxford, Oxford, UK \\ 3 Rashmi Shah Consultancy Ltd, Gerrards Cross, UK \\ 4 Proteus Associates, Oxford, UK \\ 5 Tobin Consulting LLC, Newtown Square, Pennsylvania, USA \\ 6 I3BH-Universitat Politècnica de València, Valencia, Spain
}

\section{Current Paradigms: Limitations}

Current testing for proarrhythmic potential of drugs relies on determining their effect on the QT interval of the ECG. Because QT prolongation is frequently associated with blockade of the human ether-à-go-go related gene (hERG) channel, the International Conference on Harmonization (ICH) S7B guideline ${ }^{[1]}$ recommends an hERG inhibition assay as the first proxy for identifying such potential risk. Since hERG assays are relatively cheap and rapid, pharmaceutical companies invariably rely on them for early-stage triage of new compounds. The TQT (Thorough QT) study, the backbone of clinical cardiac safety testing under ICH E14, ${ }^{[2]}$ is a blunt instrument with a poor positive predictive value. Candidate compounds suspected or shown to prolong the QT interval may be rejected out of hand or attract restrictive labelling.

However, a number of QT-prolonging drugs, such as sertindole or alfuzosin, appear in these tests to be proarrhythmic but do not necessarily lead to arrhythmias. The statistical power of laterphase clinical trials is generally insufficient to predict rare but serious proarrhythmic risks that can have huge public health and economic impacts. Regulatory and postmarketing experiences have shown that most serious adverse effects appear only when a large number of patients have been exposed over long periods of time. In addition, drugs are capable of proarrhythmic mechanisms other than those identified by the hERG channel.

Given that the major cost of developing a new drug accrues during the clinical trials phase, there would be a huge economic and clinical benefit to identifying earlier, and more accurately, those drugs that are likely to cause arrhythmias.

\section{The preDiCT Project: Insight into Mechanisms}

The preDiCT project ${ }^{[3]}$ began in June 2008, with the award of a major 3-year grant from the European Commission (EC). It is one of several projects funded by the EC, coordinated by the Virtual Physiological Human Network of Excellence. The preDiCT project is aiming to develop the computational infrastructure necessary for in silico simulation, and ultimately accurate prediction, of the impact of drug candidates on cardiac electrophysiology. This research will improve our 
understanding of the mechanisms that underpin not only the prolongation of the QT interval but also the induction of dangerous arrhythmias (such as torsade de pointes [TdP]), and propose new biomarkers and methodologies for the preclinical assessment of cardiotoxicity. In turn, this will inform the need for, and the nature of, clinical data. These simulations are done at several levels, from ionic channel and single cardiac myocyte to a full 3-D human ECG. The project is developing a Virtual Research Environment for investigating the safety (and possibly the efficacy) of drugs by simulating channel-drug interactions. A major objective has already been met - to enable the simulation of drug-induced effects on the human body surface ECG to be performed thousands of times faster than was possible at the start of the project.

The project consortium is a mix of academic and industry partners, including GlaxoSmithKline, Roche and Novartis as formal partners, with AstraZeneca, Pfizer and Johnson \& Johnson collaborating on sub-projects. The ultimate goal is wider acceptance of modelling techniques within the pharmaceutical industry and regulatory authorities.

The preDiCT project benefits from three unique strengths. First, the track record of the preDiCT team in modelling and simulation in cardiac electrophysiology. Long before the initiation of the preDiCT project, researchers at the University of Oxford had demonstrated how confidence in drug safety predictions can be increased through understanding the mechanisms, using as an example two recently approved antianginal drugs. ${ }^{[4]}$ For ivabradine, they identified the I(f) current as its target and showed that blocking this current would be safe because the reduction in pacing frequency would be only modest. With regard to ranolazine, the computations illustrated why the compound could be safe by showing that the prolongation of the repolarization time it induces would not cause early afterdepolarizations (these are believed to precede the onset of TdP). Second, the project includes several teams of biosimulation experts organized into seven Working Groups, each dedicated to specific areas of investigation, and, finally, the project has a proactive Scientific Advisory Board that includes a former Senior Clinical Assessor of the UK Medicines and Healthcare products Regulatory Agency (MHRA), one current and one former divisional director of the US FDA, clinicians and pharmaceutical industry representatives, all with expertise in drug-induced QT prolongation.

\section{Workshop Engaging Regulators and Industry}

A workshop was convened on 7 and 8 February 2011 in Oxford, bringing together the preDiCT team, regulators and industry researchers. The objectives were to discuss the feasibility of in silico modelling approaches and the achievements of the preDiCT project, and to gather their advice towards assimilation of simulation into routine regulatory activities. There were 29 participants, including representatives of the industry and regulatory authorities (Health Canada, the US FDA, the UK MHRA and the Swedish Medical Products Agency, the later two also representing the European Medicines Agency [EMA]). The FDA delegation consisted of seven senior members from different centers and divisions (via video link).

Arthur Thomas, Co-chair of the preDiCT Scientific Advisory Board, introduced the Workshop with a proposal that in silico modelling could be thought of as an additional component, together with the hERG assay and in vivo nonclinical studies, in the ICH S7B paradigm of integrated risk assessment. There was unanimous agreement among the attendees that approaches beyond a simple reliance on TQT and QT effects of a drug were necessary to predict risk.

\section{Presentations from the preDiCT Working Groups}

\section{1 State-of-the-Art Cardiac Electrophysiology Modelling and Simulation Tools}

Javier Saiz described advances in detailed modelling of drug-ion channel interaction and the validation of the models at the ionic and cellular level. New models of how different drugs (cisapride, dofetilide and lidocaine [lignocaine]) 
interact with the different states of the channels have been developed. Using these models it is possible to observe the time course of current inhibition when the drug is applied, and to reproduce the frequency-dependent effect of some drugs. For drugs that affect multiple channels, it was shown that classical approaches such as hERG inhibition assays can give rise to false positive (ebastine) and false negative (HMR1556 and JNJ 303) predictions. For these compounds, this new approach based on simulation of their effect on QT interval helped to improve prediction of their cardiotoxicity potential.

Blanca Rodriguez outlined advances used to create 3-D models of human cardiac electrical activation. The Working Group has created a highfidelity 3-D rabbit-specific electrophysiology model of the cardiac ventricles, including the specialized conduction system. Simulations using the rabbit model yield a realistic activation sequence, similar to the one recorded in experiments. Furthermore, the Working Group has produced mathematical models to simulate drug-induced effects on the human heart from the knowledge of drug-ion channel interactions and then predict their effects on the ECG (and the QT interval) measured on the surface of the 3-D human torso. The presentation stressed the importance of using only robust and tested software (such as the open-source software Chaste developed by the preDiCT team) in order to trust simulation results.

\subsection{Improving Prediction of Torsade De Pointes}

Gary Mirams presented work undertaken with project partner GlaxoSmithKline. They are working to improve the prediction of TdP risk in early stages of drug development by using mathematical models of single cardiac myocytes to integrate experimental data on drug block of three commonly measured ion channels (hERG, $\mathrm{Na}^{+}$ and L-type $\mathrm{Ca}^{++}$). Of the 31 drugs with known TdP risk, they found that risk classification as 'safe' or 'dangerous' according to hERG inhibition alone misclassified nine drugs, while examining the changes to the whole-cell action potential correctly classified all but one.
In another presentation, Blanca Rodriguez illustrated how the Working Group has combined clinical, experimental and computational investigations to improve prediction of drug cardiotoxicity and to find new biomarkers of drug cardiotoxicity. The ECG still remains the main clinical tool to detect potentially lethal drug-induced effects. Now, we can begin to investigate the relationship between the ECG and the underlying activity of the heart in silico. This will allow us to gain insight into their relationship and shed light on the 'inverse problem' of interpreting ECGs. In vivo ECG recordings from Pfizer and clinical collaborators are analysed for a variety of ECG biomarkers to determine better ways of predicting drug cardiotoxicity. Furthermore, detailed investigation of the ionic mechanisms of drug-induced arrhythmias is performed using the computational framework developed in preDiCT to inform the search for new and more specific biomarkers.

\section{Workshop Discussion}

Following these presentations, workshop participants split into breakout groups to discuss the scientific and technical aspects of future research and how this approach can be integrated into the pharmaceutical development pipeline as well as the regulatory assessment of safety.

Overall, although the regulators still had some reservations, they responded very positively and there was broad agreement that this research was in line with the priorities set by regulatory bodies, including the FDA Critical Path Initiative ${ }^{[5]}$ and the EMA Think-Tank Report on Innovative Drug Development. ${ }^{[6]}$ The FDA Initiative specifically identifies drug-induced QT prolongation as one of the areas for active research, recommending that the clinical risks associated with a small degree of QTc interval prolongation needs to be fully defined.

The regulators, as well as industry participants, identified several challenges that remain in achieving successful validation and regulatory acceptance of in silico models.

- Further development and validation of the in silico approaches would necessitate identification of specific compounds and protocols required to establish the predictive power of 
the simulations to meet the standards of the requirements of regulators and industrial partners. One difficulty foreseen is that, for many drugs, the only available ion channel data are for hERG; therefore, it may not be possible to determine whether a drug has activity at other channels. Greater collaboration with industrial partners was suggested as a way of acquiring multiple channel data.

- Safety pharmacology assays at present are not standardized in terms of cell line $(\mathrm{CHO}$ or HEK), tissue, species, pulse protocols, incubation medium, temperature, etc., and, therefore, inter-laboratory variability in results is high.

- Safety pharmacology data available from public domain sources are typically summary statistics, not detailed raw data, which limits how they can be applied.

- For many new drugs with TQT results available, postmarketing experience is currently insufficient to comment on their proarrhythmic risk, whereas for older drugs with well established risk, satisfactory interval data from clinical trials or a TQT are usually lacking.

- Raw detailed ECG public data are of limited value (e.g., data from the Cardiac Safety Research Consortium are limited to moxifloxacin).

In brief, regulators and industry would like a better understanding of the confidence with which they can rely on the project's models. To provide this, researchers require agreed protocols and detailed ion channel data on a range of compounds, as well as the level of precision desired, the choice of species, presence or absence of other variables such as ischaemia and electrolyte imbalance, experimental conditions, etc. The experimental protocols proposed will have to be accepted by all stakeholders since it is not realistic to have different protocols to meet individual requirements.

A useful preliminary step would be the identification of a list of drugs with multiple ion channel activities, for which both non-clinical and clinical data relevant to proarrhythmic safety are available, with stakeholder (academia, industry, regulatory agencies) collaboration and agreement. The selection process should ensure choices of drugs with mild, moderate and severe proarrhythmic risk, different types of proarrhythmia risk and dif- ferent mechanisms of action, in addition to drugs with a well documented lack of effect on cardiac electrophysiology. The project team was strongly encouraged to investigate the possibility of collaboration with the International Life Science Institute-Health and Environmental Sciences Institute (ILSI-HESI; www.ilsi.org), with a view to accessing their high-quality rich datasets.

Once there is consensus on a standardized experimental protocol and a list of drugs of interest, prospective high-quality experiments can proceed to characterize the effects of these drugs on a panel of multiple cardiac ion channels, including, but not limited to, hERG. Additional considerations should include definition of validation criteria and calibration of the in silico models. Ultimately, it would be necessary to determine what proarrhythmias in silico models can predict (just the TdP, or other ventricular tachyarrhythmias as well) and whether in silico models can quantify the risk in terms of severity and frequency.

Once these challenges are overcome, many potential applications were envisaged.

- Resurrecting 'good' drugs prematurely culled at the hERG assay stage. There may be a need to consider torsadogenicity in terms of patientrelated risk factors to set up profiles of 'safe' versus 'risky' drug candidates.

- During early-stage investigations, developing models in tandem with the development of new compounds, to predict safety concerns.

- Late-stage rescue (models elucidating complex mechanisms).

- Strengthening a new drug dossier intended for submission to regulatory authorities.

- New safety/regulatory approaches during evaluation of new drugs, such as use of in silico screening by the regulatory agency to investigate compounds, and/or requiring in silico investigations as part of routine filings.

- Patient stratification (identifying likely 'safe' or 'at risk' subgroups for particular drugs, especially in the context of co-morbidity and comedications).

Following this workshop, the attendees provided additional feedback via an anonymous questionnaire. Based on the feedback, the project team intends to write a proposal for future re- 
search, and to convene a follow-up meeting to discuss this proposal. Further details on this will be available from the preDiCT website. ${ }^{[3]}$

All tools and models, including the Virtual Research Environment, will be open-sourced at the end of the project in May 2011. The project team may be able to provide training or conduct additional case studies before then. If you are interested in using the models and software or would like to propose their application to a research project, please contact Katherine Fletcher. Any other comments would also be very welcome.

\section{Acknowledgements}

Supported by the EC Framework 7 programme: DG-INFSO 224381. Dr Rodriguez gratefully acknowledges the receipt of Career Development Award from the Medical Research Council.

Gary Mirams has been awarded a grant for future work from the GlaxoSmithKline Plc grants and affiliates scheme. The other authors have no conflicts of interest to declare. There was no financial assistance provided for the preparation of this report. The authors are grateful to the preDICT Steering Committee for approving the funding for free online access to this paper.

\section{References}

1. ICH Topic S7B. Note for guidance on the nonclinical evaluation of the potential for delayed ventricular repolarization (QT interval prolongation) by human pharmaceuticals (CHMP/ICH/423/02). London: European Medicines Agency, 2005 Nov [online]. Available from URL: http://www. ema.europa.eu/docs/en_GB/document_library/Scientific guideline $/ 2009 / 09 /$ WC500002841.pdf [Accessed 2011 Mar 23]

2. ICH Topic E14. Note for guidance on the clinical evaluation of QT/QTe interval prolongation and proarrhythmic potential for non-antiarrhythmic drugs (CHMP/ICH/2/04) [online]. London: European Medicines Agency, 2005 Nov. Available from URL: http://www.ema.europa.eu/docs/en GB/document library/Scientific guideline/2009/09/WC50000 2879.pdf [Accessed 2011 Mar 23]

3. The preDiCT Project [online]. Available from URL: http:// www.vph-predict.eu/ [Accessed 2011 Mar 23]
4. Rodriguez B, Burrage K, Gavaghan D, et al. The systems biology approach to drug development: application to toxicity assessment of cardiac drugs. Clin Pharmacol Ther 2010; 88: $130-4$

5. Innovation or stagnation: challenge and opportunity on the critical path to new medical products. Silver Spring (MD): US Department of Health and Human Services, Food and Drug Administration, 2004 Mar [online]. Available from URL: http://www.fda.gov/downloads/ScienceResearch/Special Topics/CriticalPathInitiative/CriticalPathOpportunitiesReports/ ucm113411.pdf [Accessed 2011 Mar 23]

6. Innovative drug development approaches: final report from the EMEA/CHMP Think-Tank Group on innovative drug development. Doc. ref. EMEA/127318/2007. London: European Medicines Agency, 2007 Mar 22 [online]. Available from URL: http://www.ema.europa.eu/docs/en_GB/ document library/Other/2009/10/WC500004913.pdf [Accessed 2011 Mar 23]

\section{Additional Reading Material}

A. Brennan T, Fink M, Rodriguez B. Multiscale modelling of drug-induced effects on cardiac electrophysiological activity. Eur J Pharm Sci 2009; 36: 62-77

B. Pueyo E, Husti Z, Hornyik T, et al. Mechanisms of ventricular rate adaptation as a predictor of arrhythmic risk. Am J Physiol Heart Circ Physiol 2010; 298: H1577-87

C. Corrias A, Jie X, Romero L, et al. Arrhythmic risk biomarkers for the assessment of drug cardiotoxicity: from experiments to computer simulations. Philos Transact A Math Phys Eng Sci 2010; 368: 3001-25

D. Mirams GR, Cui Y, Sher A, et al. Simulation of multiple ion channel block provides improved early prediction of compounds' clinical torsadogenic risk. Cardiovasc Res. Epub 2011 Mar 4

E. Zemzemi N, Bernabeu M, Saiz J, et al. Simulating druginduced effects on the heart: from ion channel to body surface electrocardiogram. Lect Notes Comput Sci. In press

F. Saiz J, Gomis-Tena J, Monserrat M, et al. Effects of antiarrhythmic drug dofetilide on transmural dispersion of repolarization in ventriculum: a computer modeling study. IEEE Trans Biomed Eng 2011; 58: 43-53

Correspondence: Mrs Katherine Fletcher, University of Oxford, Sherrington Building, Parks Road, Oxford OX1 3PT, UK.

E-mail: katherine.fletcher@dpag.ox.ac.uk 